\title{
Proinflammatory CyTOKINES Il-6 AND TNF- $\alpha$ AND THE Development of Inflammation in Obese Subjects
}

\author{
K. Popkoํㄹ E. Gorska1 , A. Stelmaszczyk-Emmel ${ }^{1}$, R. Plywaczewski², A. Stoklosa², D. Gorecka², \\ B. Pyrzak ${ }^{3}$, U. Demkow 3
}

\begin{abstract}
${ }^{1}$ Department of Laboratory Diagnostics and Clinical Immunology of Developmental Age, Warsaw Medical University, Warsaw, Poland; ${ }^{2}$ Second Clinic of Pulmonary Diseases, Institute of Tuberculosis and Lung Diseases, Warsaw, Poland; ${ }^{3}$ Clinic of Children's Endocrinology, Warsaw Medical University, Warsaw, Poland
\end{abstract}

\begin{abstract}
Background: The development of obesity and related disorders, e.g., type II diabetes (T2D), hypertension, and metabolic disturbances is strongly related to increased levels in proinflammatory cytokines (IL-1, IL6 , and TNF- $\alpha$ ). Both IL- 6 and TNF- $\alpha$ are secreted by adipocytes and their concentration correlates with the percentage and distribution of fat tissue in the body. Both cytokines are the main factors responsible for the induction of acute phase proteins production (e.g., CRP) and to inflammatory state.

Objective: To compare of TNF- $\alpha$ and IL-6 concentrations in serum from obese subjects with those in subjects with normal BMI and to analyze the relation between TNF- $\alpha$, IL-6, BMI and the inflammatory state as measured by the level of CRP.

Material and methods: The study included 80 obese subject (54 males and 26 females) BMI $>25 \mathrm{~kg} / \mathrm{m}^{2}$. A control group consisted of 53 healthy subjects (24 males and 29 females) with BMI $<25 \mathrm{~kg} / \mathrm{m}^{2}$. To determine the blood plasma concentration of IL-6 and TNF, commercial ELISA assay kits were used.

Results: The concentration of IL-6 was lower in the control compared with the obese patients, but a significance difference concerned only female subjects $(\mathrm{P}=0.001)$. TNF- $\alpha$ concentration was significantly higher in all obese subjects $(\mathrm{P}<0.001)$. A higher level of this cytokine was also found in patients with obesity suffering from T2DM. A positive correlation was present between IL6 and TNF- $\alpha$ concentrations. Only did the IL- 6 level correlate with the concentration of CRP in serum.

Conclusions: The study confirmed that increased inflammatory cytokines lead to the persistence of inflammation in obese subjects. However, some other factors, such as gender, may contribute to the development of obesity-related inflammatory states.
\end{abstract}

Key words: obesity, inflammatory cytokines, TNF, IL6, CRP

\section{INTRODUCTION}

Proinflammatory cytokines (IL-1, IL-6, and TNF- $\alpha$ ) may significantly influence the development of obesity and concomitant disorders such as type 2 diabetes, arterial hypertension and metabolic disorders. These cy- tokines substantially regulate energy balance and lipid and carbohydrates metabolism [1,2]. They are expressed in subcutaneous and intraperitoneal adipocytes [3].

IL-6 is one of major proinflammatory cytokines responsible for immune response activation. Its pleiotropic activity includes acute phase proteins synthesis in liver and lymphocyte differentiation [4]. It is also able to influence hormonal balance and some endocrinological abnormalities. It is suggested, that IL-6 may affect the increase of free fatty acids level. IL-6 concentration is elevated in the patients with lipid abnormalities and insulin resistance [5].

TNF- $\alpha$ is secreted mainly by macrophages and lymphocytes in response to cell damage caused by infection or malignant transformation [6]. However, it can be also secreted by many other type of cells and tissues, e.g., adipocytes [7]. TNF- $\alpha$, similarly to IL- 6 , is a proinflammatory cytokine, characterized by a broad spectrum of functions which also include cytotoxic and cytostatic effects against cancer cells [8].

TNF- $\alpha$ exerts an important influence on adipose tissue metabolism and function. It is able to inhibit lipoprotein lipase (LPL) at both mRNA and protein levels. It also inhibits the expression of two major adipose tissue differentiation regulators: CCAAT transcription factor that increases binding of alpha proteins (CEBP- $\alpha$ ) and nuclear receptor PPAR $\gamma$-2. In consequence, changes in the expression of adipose tissue proteins, such as LPL, AP2, fatty acid synthase, acetyl-coA carboxylase, glycerol 3-phosphate dehydrogenase (GPDH), glucose receptor GLUT-4, and others are observed [9]. Moreover, TNF- $\alpha$ and IL-6 are able to influence each other's secretion level.

The aim of the present study was to assess the relation between IL- 6 and TNF- $\alpha$ levels in serum of obese and normal weight subjects and to relate it to the CRP concentration in obese subjects.

\section{MATERIAL AND METHODS}

The study was performed in accordance with the Declaration of Helsinki for Human Research and study protocol was approved by the Ethics Committee of Warsaw Medical University in Warsaw, Poland.

The study included 80 obese subjects with body mass index $(\mathrm{BMI})>25 \mathrm{~kg} / \mathrm{m}^{2}$, including 54 males aged 
31-77 (mean $53 \pm 1 S E$ years) and 26 females aged 3975 (mean $61 \pm 2$ years). A control group included 53 healthy subjects with normal weight, BMI $<25 ; 24$ males aged $22-72$ (mean $39 \pm 2.3$ years) and 29 females aged $23-78$ (mean $47 \pm 2.4$ years). In the studied population no allergic, hematologic, or other immunologic diseases were found. The tests were performed in a disease-free period, at least 3 weeks after any last infection, antibiotic treatment, and at least 3 months after any severe disease.

Blood samples were collected using EDTA as anticoagulant. To determine blood plasma concentration of IL-6 and TNF, 'Quantikine human IL-6' and 'Quantikine human TNF' (R\&D Systems, MN, USA) ELISA assay kits, respectively, were used. The measurements were taken using a Microplate Reader Stat Fax 2100 (Awareness Technology, USA). CRP plasma concentration was determined with fixed-point immuno-rate method (Ortho-Clinical-Diagnostics).

Results were analyzed for the whole population as well as in relation to gender, and compared with the control group. Mean cytokine levels in different groups were compared with the Mann-Whitney U test. Correlations between the examined parameters were assessed using Pearson's or Spearman's test. $\mathrm{P}<0.05$ was accepted as indicative of statistical significant. Calculations were performed using STATISTICA software and Microsoft Excel.

\section{Results}

The mean IL-6 level was generally lower in the control group in comparison with the population of patients population. However, a statistically significant difference in IL-6 was found only between obese and normal weight women $(\mathrm{P}=0.001)$, and not in males. TNF secretion in the obese patients was significantly higher than in the control group. The highest difference was found again with respect to the group of women $(\mathrm{P}<0.001)$.

In addition, IL-6 level was higher in the obese patients with type 2 diabetes in comparison with the non-diabetic obese patients. Here again, a statistically significant difference was observed only in the group of women $(\mathrm{P}=0.01)$. The obese diabetic patients had a slightly higher TNF concentration than the obese non-diabetic patients; the highest, albeit insignificant, difference being in the group of obese women.

Statistical analysis showed a positive correlation between IL-6 and TNF concentrations; significant values were achieved for the whole groups of overweight and obese patients $(\mathrm{P}=0.005)$ (Fig. 1$)$. In whole populations, regardless of gender, a simultaneous elevation of both proinflammatory cytokines was observed.

The CRP level positively correlated with the IL-6 concentration. Here, the results for the whole overweight and obese group as well as in the women group were statistically significant. This correlation in the group of men was insignificant (Fig. 2). No statistically significant correlation between TNF and CRP concentrations in overweight and obese patients was evident.

The CRP level significantly correlated with the obesity index expressed by BMI; the correlation was observed in all analyzed subgroups (Fig. 3).

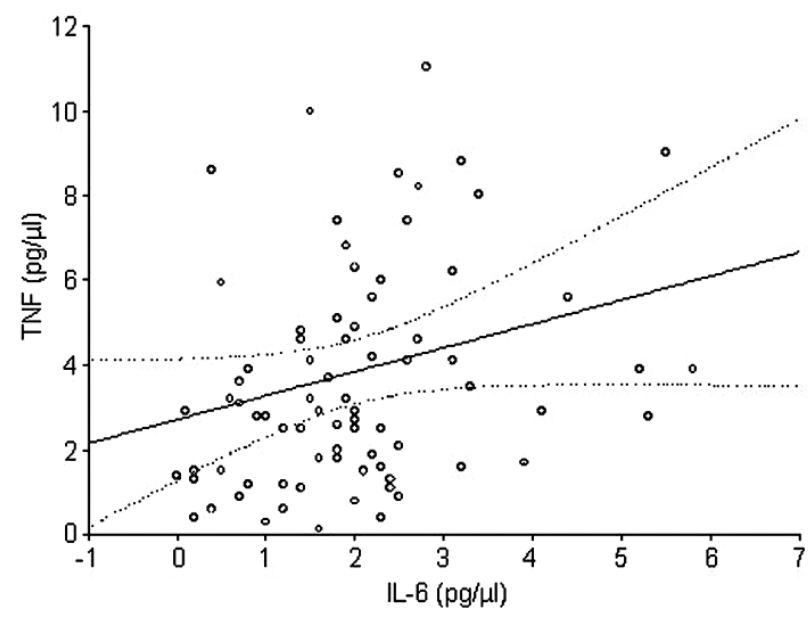

Fig. 1. Correlation between serum concentrations of IL-6 and $\operatorname{TNF}(r=0.3 ; \mathrm{P}=0.005)$.

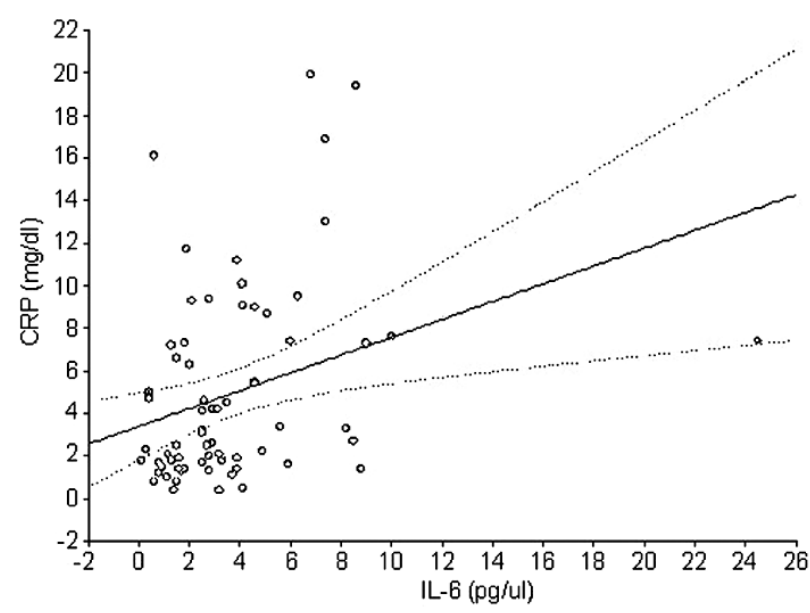

Fig 2. Correlation between IL-6 concentration and CRP level in the population of overweight and obese patients $(\mathrm{r}=0.3$; P $=0.007)$.

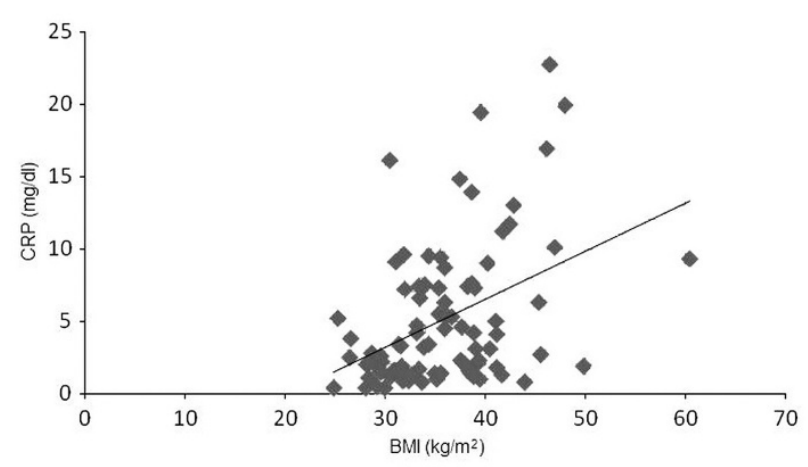

Fig. 3. Correlation between BMI values and CRP levels in the population of overweight and obese patients $(r=0.4$; $\mathrm{P}<0.001)$.

\section{Discussion}

It is widely suggested that overweight and obese subjects have elevated concentrations of inflammatory cytokines in serum. A possible mechanism of obesity-induced inflammatory response is based on the develop- 
ment of oxidative stress which, in consequence, leads to the inflammatory response induced by excessive intake of carbohydrates, mainly glucose. Recent studies have shown that up to $30 \%$ of IL-6 in the blood stream is secreted by adipose tissue, and the cytokine level positively correlates with body mass index (BMI) [10]. Intraperitoneal adipose tissue secretes three times more of IL-6 than does subcutaneous adipose tissue [3]. In the present study, we have observed a significantly higher IL-6 concentration in obese women. Moreover, a difference was observed in the population of obese, diabetic women in comparison with the women with no symptoms of diabetes. The obtained results suggest that gender is a major factor influencing the regulation of inflammatory factors; possibly due to a specific hormonal balance or adipose tissue distribution in women.

Some authors consider both type 2 diabetes and insulin resistance as symptoms of an inflammatory response in the organism [5]. The hypothesis can be confirmed by the increase in acute phase proteins, such as CRP, amyloid A, alpha-1-acid glycoprotein, sialic acid, and cortisone in diabetic patients [11]. Moreover, increases in proinflammatory cytokines are found in the obese population. The increase in IL-6, parallel to the elevated glucose and insulin concentrations, suggest a possible involvement of the cytokine in glucose metabolism, especially in adipocytes. Likewise, in the present study we observed that cytokines positively correlated with the CRP level, while CRP, in turn, positively correlated with BMI in all analyzed subpopulations of obese patients.

Obesity is often associated with insulin resistance and hyperinsulinemia. The increase in TNF expression in muscles and adipose tissue, through the TNF ability to phosphorylate serine residues in IRS-I (insulin receptor substrate 1) and a consequent decrease of insulin receptor tyrosine kinase activity, can lead to insulin resistance [12]. The increase of TNF expression in the obese patients definitely demonstrated in the present study, can be a limiting factor for further enlargement of adipose tissue through the induction of insulin resistance of adipocytes [13]. Insulin resistance can influence lipoprotein lipase (LPL) activity and hence prevent excessive growth of adipose tissue. A high adipose tissue LPL to muscle LPL ratio observed in hyperinsulinemia leads to increased transport of lipids to the adipose tissue [14]. So, rising insulin resistance will cause the reversal of the adipose tissue LPL to muscle LPL ratio, and in that way prevent further growth of adipose tissue [15]. We suppose that TNFinduced insulin resistance, hyperinsulinemia, and higher LPL activity in adipocytes can cause body fat accumulation. On the other hand, the increase in body fat may lead to increased secretion of TNF that, in consequence, can cause insulin resistance [16]. It is hard to state unambiguously whether the body fat content is a cause or a consequence of the observed differences in TNF concentration. However, it seems that the development type 2 diabetes depends on a number of other factors being more potent that TNF, as we did not find any differences in the TNF levels between the obese diabetics and non-obese patients with no diabetes.
Conflicts of interest: The authors declare no conflict of interest in relation to this article.

\section{REFERENCES}

1. Fruhbeck G, Gomez-Ambrosi J, Muruzabal FJ, Burrel MA. The adipocyte: a model for integration of endocrine and metabolic signaling in energy metabolism regulation. Am J Physiol Endocrinol Metab 2001; 280: 827-47.

2. Grunfeld C, Feingold KR. The metabolic effects of tumor necrosis factor and other cytokines. Biotherapy 1991; 3: 143-58.

3. Fried SK, Bunkin DA, Greengerg AS. Omental and subcutaneous adipose tissue of obese subjects release interleukin-6: depot difference and regulation by glucocorticoid. J Clin Endocrinol Metab 1998; 83: 847-50.

4. May LT, Ghrayeb J, Santhanam U, Tatter SB, Sthoeger Z, Helfgott DC, Chiorazzi N, Grieninger G, Sehgal PB. Synthesis and secretion of multiple forms of beta 2-interferon/B-cell differentiation factor $2 /$ hepatocyte-stimulating factor by human fibroblasts and monocytes. J Biol Chem 1988; 263: 7760-6.

5. Pickup JC, Mattock MB, Chusney GD, Burt D. NIDDM as a disease of the innate immune system: association of acute-phase reactant and interleukin-6 with metabolic syndrome X. Diabetologia 1997; 40: 1286-92.

6. Beutler B, Cerami A. The biology of cachectin/TNF- $\alpha$ primary mediator of the host response. Annu Rev Immunol 1989; 7: 625-55.

7. Hotamisligil G.S, Shargill NS, Spiegelman BM. Adipose expression of tumor necrosis factor-alpha: direct role in obesity-linked insulin resistance. Science 1993; 259: 8791.

8. Vassali P. The pathophysiology of tumor necrosis factor. Annu Rev Immunol 1992; 10: 411-52.

9. Stephens JM, Pekala PH. Transcriptional repression of the C/EBP-alpha and GLUT4 genes in 3T3-L1 adipocytes by tumor necrosis factor-alpha. Regulations are coordinate and independent of protein synthesis. J Biol Chem 1992; 267: 13580-4.

10. Mohamed-Ali V, Goodrick S, Rawesh A, Katz DR, Miles JM, Yudkin JS, Klein S, Coppack SW. Subcutanous adipose tissue releases interleukin-6, but not tumor necrosis factor- $\alpha$, in vitro. J Clin Endocrinol Metab 1997; 82: 4196-200

11. Jonsson A, Wales JK. Blood glycoprotein levels in diabetes mellitus. Diabetologia 1976; 12: 245-50.

12. Hotamisligil GS, Peraldi P, Budavari A, Ellis R, White MF, Spiegelman BM. IRS-1-mediated inhibition of insulin receptor tyrosine kinase activity in TNF- $\alpha$ and obesity-induced insulin resistance. Science 1996; 271: 665-8.

13. Eckel RH. Insulin resistance: an adaptation for weight maintenance. Lancet 1992; 340: 1452-3.

14. Cryer A. Comparative biochemistry and physiology of lipoprotein lipase. In: Borensztajn J (ed.) Lipoprotein lipase Evener: Chicago, IL, 1987; pp 277-327.

15. Kern PA, Sadhizadeh M, Ong JM, Bosch RJ, Deem R, Simsolo RB. The expression of tumor necrosis factor in human adipose tissue. Regulation by obesity, weight loss and relationship to lipoprotein lipase. J Clin Invest 1995; 95: 2111-9.

Address for correspondence:

Katarzyna Popko

24, Marszalkowska St.

00-576 Warsaw

Poland

Phone/fax: +48 226296517

E-mail: aksiak@interia.pl 